\title{
Laxminarayan Sahoo
}

\section{Method for solving intuitionistic fuzzy assignment problem}

\begin{abstract}
The method to find an answer for assignment problem (AP) under intuitionistic fuzzy domain is proposed in this chapter. Due to the irregular rising and falling of the present market economy, here we have assumed that the assignment costs are not always fixed. Therefore, the assignment costs are imprecise in nature. In the existing literature, different approaches have been used, which are interval, fuzzy, stochastic, and fuzzy-stochastic approaches to represent the impreciseness. In this chapter, we have represented impreciseness taking intuitionistic fuzzy numbers (IFN). The proposed method is hinged on ranking of IFN and use of wellknown Hungarian method. Here, we have used a newly proposed centroid concept ranking method for IFNs. In this chapter, we have solved AP where costs for assignment are taken as triangular IFNs. A numerical example has been considered to derive the optimal result and also to adorn the applicability of the suggested method. In the end, concluding remarks and future research of the proposed approach have been presented.
\end{abstract}

Keywords: assignment problem, intuitionistic fuzzy numbers, Hungarian method, impreciseness, triangular intuitionistic fuzzy numbers

\subsection{Introduction}

Assignment problem (AP) is effectively used in solving realistic problems for finding the best alternative solutions. AP plays an essential role in managerial decisionmaking. It is related to the problem of production planning, scheduling, and engineering design problem. The basic task of AP is to allocate many tasks/jobs in a more efficient way such that ideal assignment can be obtained in an acceptable limit. In an AP, $n$ workers and $n$ jobs and effectiveness of each worker for each job, the problem involves in assigning each worker to only one job so that the total amount of effectiveness is optimized. In reality, different procedures and algorithms, namely, linear programming method [1-3], Hungarian algorithm [4], neural network method [5], genetic algorithm [6], and so on were used to solve the AP. In traditional AP, it is assumed that all the parameters related to AP are fixed valued. Notwithstanding in reality, these parameters are not fixed due to uncertainty and lack of proper information. The uncertainty may occur resulting from some factors: (i) decision maker has

Laxminarayan Sahoo, Department of Computer and Information Science, Raiganj, West Bengal, India

Ә Open Access. ( 2020 Laxminarayan Sahoo, published by De Gruyter. (c) BY-NC-ND This work is licensed under a Creative Commons Attribution-NonCommercial-NoDerivatives 4.0 International License.

https://doi.org/10.1515/9783110628616-008 
no sense about the assignment parameters when a job is to be scheduled at the beginning time, as a result some uncertainty may occur with regard to assignment parameters; (ii) nowadays present market economy is always flooded, that is, always unstable due to competing market and, as a consequence, the assignment cost is totally changeable. To succeed in dealing with these types of condition, the problem can be framed using the notion of uncertainty and parameters are served as imprecise/uncertain in nature. In such possible situations, fuzzy set (FS) theory plays an important role in picking up such situation. The theory of FS was originated by Zadeh [7] in 1965 and it dealt with imprecision and vagueness in real-world situations. In the year 1970, Belmann and Zadeh [8] introduced the notion of decisionmaking problems assuming uncertainty. Here, we have served imprecise parameters considering fuzzy numbers. Therefore, the fuzzy AP (FAP) yields an efficient framework that solves real-life problems with uncertain information. In the last few years, several efforts have been made in the existing relevant works for solving FAP. A number of researchers [9-15] have formulated AP in different sectors/areas such as firm management, vehicle routing, and network management considering fuzzy parameters and/or interval parameters. For solving these problems, they have also used several mathematical techniques/methods like linear programming technique and genetic algorithm. Chen and Chen [16] described about fuzzy optimal solution of the AP based on the ranking of generalized fuzzy numbers. Fuzzy risk analysis based on ranking fuzzy numbers using $\alpha$-cuts was proposed by Chen and Wang. Rommelfanger [17] discussed a ranking of fuzzy cost present in the FAP, which takes more advantages over the existing fuzzy ranking methods. Kar et al. [18] unfolded the fuzzy generalized AP with restriction on available cost. Solution of FAP with ranking of generalized trapezoidal fuzzy numbers was resolved by Thangavelu et al. [19]. Andal et al. [20] discussed a technique to minimize cost flow of FAP considering fuzzy membership. Pramanik and Biswas [21] introduced multiobjective AP with generalized trapezoidal fuzzy numbers. Mukherjee and Basu [22] suggested the application of fuzzy ranking method for solving AP with fuzzy cost. Very recently, Sahoo and Ghosh [23] solved AP with linguistic costs.

In fuzzy optimization technique, the degrees of receiving objective functions and constraints are considered. FS theory has also been evolved in several fields and its different modifications/upgradations have come to light. The most important generalizations of FS theory is an intuitionistic FS (IFS) which was originated by Atanassov [24] in 1986. The concept of IFS is an alternative approach to define FS in the case where information is not available sufficiently for defining impreciseness by means of ordinary FS. An IFS is characterized by the degree of acceptance and degree of rejection. The IFS theory has been applied in different fields including decision-making. For more details, one may refer to the works of Jana and Roy [25], Roy et al. [26], and Kumar and Hussian [27].

In this chapter, we have considered intuitionistic FAP (IFAP). To solve the IFAP, we have used a new ranking method based on centroid concept introduced by Arun 
Prakash et al. [28]. Then the AP has been remodeled into an AP whose parameters are fixed/crisp valued and solved by linear programming method and/or Hungarian method [4]. An example has been studied to obtain the optimal result and also to illustrate the applicability of the proposed method. The rest of the chapter is presented as follows:

In Section 8.2, brief notions about IFSs and fuzzy numbers are described. Mathematical formulation of AP is given in Section 8.3. Section 8.4 gives the solution methodology of the IFAP. In Section 8.5, a numerical example is given for illustration purpose. Section 8.6 concludes the chapter with further scope of research.

\subsection{Preliminaries}

In this section, a brief idea about IFSs and fuzzy numbers used in this chapter is presented.

Definition 8.1 ([23]). Let $X$ be a given nonempty set. An IFS $\tilde{A}$ in $X$ is defined by $\tilde{A}=\left\{\left(x, \mu_{\tilde{A}}(x), \gamma_{\tilde{A}}(x)\right): x \in X\right\}$ where $\mu_{\tilde{A}}(x): X \rightarrow[0,1]$ and $\gamma_{\tilde{A}}(x): X \rightarrow[0,1]$ define, respectively, the degree of acceptance and degree of rejection of the element $x \in X$ to the set $\tilde{A}$ which is a subset of $X$ and every $x \in X, 0 \leq \mu_{\tilde{A}}(x)+\gamma_{\tilde{A}}(x) \leq 1$. Again each IFS $\tilde{A}$ in $X$, the function $\pi_{\tilde{A}}(x)=1-\mu_{\tilde{A}}(x)-\gamma_{\tilde{A}}(x)$ is called the degree of hesitation. If $\pi_{\tilde{A}}(x)=0$ for all $x \in X$ then IFS becomes FS.

Definition 8.2. An IFS $\tilde{A}$ is called normal if there exists $x_{0} \in X$ such that $\mu_{\tilde{A}}\left(x_{0}\right)=1$ and $\gamma_{\tilde{A}}\left(x_{0}\right)=0$.

Definition 8.3. An IFS $\tilde{A}$ is called convex if for all $x_{1}, x_{2} \in X$ and $0 \leq \lambda \leq 1 \mu_{\tilde{A}}$ $\left(\lambda x_{1}+(1-\lambda) x_{2}\right) \geq \operatorname{Min}\left\{\mu_{\tilde{A}}\left(x_{1}\right), \mu_{\tilde{A}}\left(x_{2}\right)\right\}$ and $\gamma_{\tilde{A}}\left(\lambda x_{1}+(1-\lambda) x_{2}\right) \geq \operatorname{Min}\left\{\gamma_{\tilde{A}}\left(x_{1}\right), \gamma_{\tilde{A}}\left(x_{2}\right)\right\}$.

Definition 8.4. Support of IFS $\tilde{A}$ with universal set $X$ is denoted by Support $(\tilde{A})$ and is defined by Support $(\tilde{A})=\left\{x: \mu_{\tilde{A}}(x)>0\right.$ and $\left.\gamma_{\tilde{A}}(x) \leq 1, x \in X\right\}$.

Definition 8.5. An IFS $\tilde{A}=\left\{\left(x, \mu_{\tilde{A}}(x), \gamma_{\tilde{A}}(x)\right): x \in X\right\}$ of the real line is called an IFN if

(i) $\tilde{A}$ is intuitionistic fuzzy (IF) normal;

(ii) $\tilde{A}$ is IF convex;

(iii) $\mu_{\tilde{A}}(x)$ is upper semicontinuous and $\gamma_{\tilde{A}}(x)$ is lower semicontinuous; and

(iv) $\operatorname{Support}(\tilde{A})$ is bounded. 
Definition 8.6. An IFN $\tilde{A}=\left(a_{1}, a_{2} . a_{3}\right)\left(\bar{a}_{1}, a_{2}, \bar{a}_{3}\right)$ where $\bar{a}_{1} \leq a_{1} \leq a_{2} \leq a_{3} \leq \bar{a}_{3}$ is said to be triangular IFN if its membership and nonmembership functions, respectively, are defined as follows:

$$
\mu_{\tilde{A}}(x)= \begin{cases}\frac{x-a_{1}}{a_{2}-a_{1}} & \text { if } a_{1} \leq x \leq a_{2} \\ \frac{a_{3}-x}{a_{3}-a_{2}} & \text { if } a_{2} \leq x \leq a_{3} \\ 0 & \text { otherwise }\end{cases}
$$

and

$$
\gamma_{\tilde{A}}(x)= \begin{cases}\frac{a_{2}-x}{a_{2}-a_{1}} & \text { if } \bar{a}_{1} \leq x \leq a_{2} \\ \frac{x-a_{2}}{\bar{a}_{3}-a_{2}} & \text { if } a_{2} \leq x \leq \bar{a}_{3} \\ 1 & \text { otherwise }\end{cases}
$$

Definition 8.7 ([27]). The centroid point $(\tilde{x}(A), \tilde{y}(A))$ of triangular IFN $\tilde{A}=\left(a_{1}, a_{2} \cdot a_{3}\right)$ $\left(\bar{a}_{1}, a_{2}, \bar{a}_{3}\right)$ can be determined by

$$
\begin{aligned}
& x_{\mu}(A)=\frac{a_{1}+a_{2}+a_{3}}{3} \\
& x_{\gamma}(A)=\frac{2 \bar{a}_{1}-a_{2}+\bar{a}_{3}}{3} \\
& y_{\mu}(A)=\frac{1}{3}
\end{aligned}
$$

and

$$
y_{\gamma}(A)=\frac{2}{3}
$$

Definition 8.8 ([27]). The ranking function of the triangular IFN $\tilde{A}$ is defined by

$$
\rho(\tilde{A})=\left(\frac{1}{2}\left(x_{\mu}(A)-y_{\mu}(A)\right)^{2}+\frac{1}{2}\left(x_{\gamma}(A)-y_{\gamma}(A)\right)^{2}\right)^{\frac{1}{2}} .
$$

Theorem 8.1. Let $\tilde{A}$ and $\tilde{B}$ be two triangular IFNs then $\rho(\tilde{A}+\tilde{C})>\rho(\tilde{B}+\tilde{C}) \Rightarrow$ $\tilde{A}+\tilde{C} \geq \tilde{B}+\tilde{C}$.

Proof. $\quad \rho(\tilde{A}+\tilde{B})=\rho(\tilde{A})+\rho(\tilde{B})$ and $\rho(\tilde{B}+\tilde{C})=\rho(\tilde{B})+\rho(\tilde{C})$

Now if, $\tilde{A} \geq \tilde{B}$ then $\rho(\tilde{A}+\tilde{C})>\rho(\tilde{B}+\tilde{C}) \Rightarrow \tilde{A}+\tilde{C} \geq \tilde{B}+\tilde{C}$.

Theorem 8.2. Let $\tilde{A}$ and $\tilde{B}$ be two triangular IFNs, then,

$\tilde{A}<\tilde{B}$ if $\rho(\tilde{A})<\rho(\tilde{B})$

$\tilde{A}>\tilde{B}$ if $\rho(\tilde{A})>\rho(\tilde{B})$

$\tilde{A}=\tilde{B}$ if $\rho(\tilde{A})=\rho(\tilde{B})$ 


\subsection{Mathematical formulation of assignment problem}

Suppose there are $n$ jobs to be performed and $n$ workers/machines are available for doing these jobs. Here we have assumed that each worker/machine can do each job at a time, though with swing degree of efficiency. Let $c_{i j}$ be the cost or time if the $i$ th person/machine is assigned the $j$ th job, the problem is to find an assignment so that the entire cost of payment or time for performing all jobs are minimum.

Mathematically, AP can be modeled as follows:

$$
\text { Minimize } z=\sum_{i=1}^{n} \sum_{j=1}^{n} c_{i j} x_{i j}, \quad(i, j=1,2, \ldots, n)
$$

Subject to $x_{i j}=\left\{\begin{array}{l}1 \text { if } i \text { th worker } / \text { machine is assigned } j \text { th job } \\ 0 \text { if } i \text { th worker } / \text { machine is not assigned } j \text { th job }\end{array}\right.$

$$
\sum_{j=1}^{n} x_{i j}=1, i=1(1) n
$$

and

$$
\sum_{i=1}^{n} x_{i j}=1, j=1(1) n
$$

where $x_{i j}$ denotes that $j$ th job is to be assigned to the $i$ th worker/machine.

In most of the cases, it is observed that the cost of payment/time of assignment is not precise due to the fluctuation of market economy and, hence, we have assumed that the assignment costs/times are imprecise and have also considered that they are fuzzy numbers $\tilde{c}_{i j}(i=1(1) n$ and $j=1(1) n)$. Then AP (8.1) becomes FAP, which can be written as follows:

$$
\text { Minimize } \tilde{z}=\sum_{i=1}^{n} \sum_{j=1}^{n} \tilde{c}_{i j} x_{i j},(i, j=1,2, \ldots, n)
$$

Subject to $x_{i j}=\left\{\begin{array}{l}1 \text { if } i \text { th worker } / \text { machine is assigned } j \text { th job } \\ 0 \text { if } i \text { th worker } / \text { machine is not assigned } j \text { th job }\end{array}\right.$

$$
\sum_{j=1}^{n} x_{i j}=1, i=1(1) n
$$

and

$$
\sum_{i=1}^{n} x_{i j}=1, j=1(1) n
$$

where $x_{i j}$ denotes that $j$ th job is to be assigned to the $i$ th person/machine. 
Theorem 8.3. If $x_{i j}=X_{i j}$ minimizes $\tilde{z}=\sum_{i=1}^{n} \sum_{j=1}^{n} \tilde{c}_{i j} x_{i j}$ over all $x_{i j}=0$ or 1 such that $\sum_{j=1}^{n} x_{i j}=1, \sum_{j=1}^{n} x_{i j}=1$ then $x_{i j}=X_{i j}$ also minimizes $\tilde{z}^{\prime}=\sum_{i=1}^{n} \sum_{j=1}^{n} \tilde{c}_{i j}^{\prime} x_{i j}$ where $\tilde{c}_{i j}^{\prime}=\tilde{c}_{i j}-\tilde{u}_{i}-\tilde{v}_{j}$

Proof. Let $x_{n} x_{i j}=X_{i j}$ minimizes the total cost $\tilde{z}=\sum_{i=1}^{n} \sum_{j=1}^{n} \tilde{c}_{i j} x_{i j}$ over all $x_{i j}=0$ or 1 and $\sum_{j=1}^{n} x_{i j}=1, \sum_{j=1}^{n} x_{i j}=1$.

Now it is to be shown that $x_{i j}=X_{i j}$ also minimizes a new cost $\tilde{z}^{\prime}=\sum_{i=1}^{n} \sum_{j=1}^{n}\left(\tilde{c}_{i j}-\tilde{u}_{i}-\tilde{v}_{j}\right)$ $x_{i j}$ where $\tilde{u}_{i}$ and $\tilde{v}_{j}$ are imprecise cost constants subtracted from cost matrix $\left(\tilde{c}_{i j}\right)_{n \times n}$.

Again $\quad \tilde{z}^{\prime}=\sum_{i=1}^{n} \sum_{j=1}^{n} \tilde{c}_{i j} x_{i j} \sum_{j=1}^{n} \tilde{c}_{i j} x_{i j}-\sum_{i=1}^{n} \sum_{j=1}^{n} \tilde{u}_{i} x_{i j}-\sum_{i=1}^{n} \sum_{j=1}^{n} \tilde{v}_{j} x_{i j}=z-\sum_{i=1}^{n} \tilde{u}_{i} \sum_{j=1}^{n} x_{i j}-\sum_{i=1}^{n} x_{i j} \sum_{j=1}^{n} \tilde{v}_{j}=$ $z-\sum_{i=1}^{n} \tilde{u}_{i}-\sum_{j=1}^{n} \tilde{v}_{j}$

So, here $\tilde{z}^{\prime}$ is independent of $x_{i j}$ 's, and hence $\tilde{z}^{\prime}$ is minimized when $\tilde{z}$ is minimized and vice versa.

Theorem 8.4. In an AP with cost matrix $\left(\tilde{c}_{i j}\right)_{n \times n}$, if all $\tilde{c}_{i j} \geq \tilde{0}$ then a feasible solution $x_{i j}$ is optimal when $\sum_{i=1}^{n} \sum_{j=1}^{n} \tilde{c}_{i j} x_{i j}=\tilde{0}$.

Proof. Since all $\tilde{c}_{i j} \geq \tilde{0}$ and all $x_{i j} \geq 0$, therefore $\left(\tilde{z}=\sum_{i=1}^{n} \sum_{j=1}^{n} \tilde{c}_{i j} x_{i j}\right) \geq \tilde{0}$. Hence, the minimum possible value of $\tilde{z}=\sum_{i=1}^{n} \sum_{j=1}^{n} \tilde{c}_{i j} x_{i j}$ can attain is always $\tilde{0}$. Then any feasible solution $x_{i j}$ is optimal iff $\sum_{i=1}^{n} \sum_{j=1}^{n} \tilde{c}_{i j} x_{i j}=\tilde{0}$.

\subsubsection{The Hungarian method}

The following method applies Theorem 8.4 to a given $n \times n$ cost matrix to obtain an optimal assignment [4].

Step 1: For each row $i, i=1,2, \ldots, n$, let $c_{i r}, r \in\{1,2, \ldots, n\}$ be the smallest entry and calculate $c_{i j}-c_{i r}$, for each $i$ and $r \in\{1,2, \ldots, n\}$.

Obviously $\operatorname{Min}\left\{c_{i j}-c_{i r}\right\}=0$, for each $i$ and $r \in\{1,2, \ldots, n\}$.

Step 2: For each column $j, j=1,2, \ldots, n$, let $c_{k j}, k \in\{1,2, \ldots, n\}$ be the smallest entry and calculate $c_{i j}-c_{k j}$, for each $j$ and $k \in\{1,2, \ldots, n\}$.

Obviously $\operatorname{Min}\left\{c_{i j}-c_{k j}\right\}=0$, for each $j$ and $k \in\{1,2, \ldots, n\}$.

Step 3: Find minimum number of lines $l$ (say) in such a way that $l 1$ ines covered all the zero entries of the cost matrix.

Step 4: Now, either $l=n$ or $l<n$. If $l=n$, then an optimal assignment is arrived. (ii) If $l<n$ then go to Step 5 .

Step 5: Find the smallest entry not covered by any line, subtract this from each uncovered row and column, and then add it to junction entry and go to Step 3. 


\subsection{Solution methodology}

Suggested approach of solution procedure of linguistic AP is as given below:

Step 1: Find $\rho\left(\tilde{c}_{i j}\right)$ of each $\tilde{c}_{i j}$ using ranking function defined in Definition 8.8.

Step 2: Check whether the given AP is balanced or not.

(i) If the AP is a balanced then go to Step 4.

(ii) If the AP is unbalanced then go to Step 3.

Step 3: Make dummy rows and/or columns with zero cost so as to form a balanced AP.

Step 4: Solved by method A: (existing linear programming problem method) and/ or by method B: (the Hungarian method described in Section 8.3.3) to obtain the appropriate assignments.

$$
\begin{array}{ll}
\text { Method A: Solve } & \text { Minimize } z=\sum_{i=1}^{n} \sum_{j=1}^{n} \rho\left(\tilde{c}_{i j}\right) x_{i j} \\
& \text { subject to } \sum_{i=1}^{n} x_{i j}=1, j=1,2, \ldots, n \\
& \sum_{j=1}^{n} x_{i j}=1, i=1,2, \ldots, n \\
& x_{i j} \in\{0,1\}
\end{array}
$$

Method B: Find an optimal assignment using Hungarian algorithm.

\subsection{Numerical example}

In this section, we have considered an IFAP with three resources $W_{1}, W_{2}, W_{3}$ and three jobs $J_{1}, J_{2}, J_{3}$ with IF assignment cost. The cost matrix $\left(\tilde{c}_{i j}\right)_{3 \times 3}$ is given below whose elements are triangular IFNs.

\begin{tabular}{lllll}
\hline \multicolumn{5}{c}{ Activities } \\
\cline { 3 - 5 } & & \multicolumn{3}{c}{$\boldsymbol{J}_{2}$} \\
\hline Resource & $W_{1}$ & $(4,7,14)(3,7,24)$ & $(12,14,16)(11,14,28)$ & $(8,15,25)(7,15,30)$ \\
& $W_{2}$ & $(6,18,25)(5,18,28)$ & $(13,19,28)(12,19,36)$ & $(20,25,33)(19,25,38)$ \\
& $W_{3}$ & $(20,25,38)(19,25,42)$ & $(2,11,14)(1,11,19)$ & $(6,12,15)(5,12,20)$ \\
\hline
\end{tabular}

Solution: Here, it is seen that the IFAP is a balanced one. Now, we have obtained $R\left(\tilde{c}_{i j}\right)$ of each $\tilde{c}_{i j}$ using ranking function mentioned in Definition 8.8. Now the reduced problem is as follows: 


\begin{tabular}{lrrrr}
\hline & & \multicolumn{3}{c}{ Activities } \\
\cline { 3 - 5 } & & $\boldsymbol{J}_{\mathbf{1}}$ & $\boldsymbol{J}_{\mathbf{2}}$ & $\boldsymbol{J}_{3}$ \\
\hline Resource & $W_{1}$ & 12.02 & 15.57 & 17.41 \\
& $W_{2}$ & 15.67 & 22.49 & 27.38 \\
& $W_{3}$ & 29.58 & 8.33 & 11.35 \\
\hline
\end{tabular}

Then, the AP can be modeled in the following linear programming problem as follows:

$$
\text { Minimize } \begin{aligned}
z= & 12.02 x_{11}+15.67 x_{21}+29.58 x_{31}+15.57 x_{12}+22.49 x_{22}+8.33 x_{32} \\
& +17.41 x_{13}+27.38 x_{23}+11.35 x_{33}
\end{aligned}
$$

Subject to

$$
\begin{gathered}
x_{11}+x_{21}+x_{31}=1 ; x_{12}+x_{22}+x_{32}=1 ; x_{13}+x_{23}+x_{33}=1 ; \\
x_{11}+x_{12}+x_{13}=1 ; x_{21}+x_{22}+x_{23}=1 ; x_{31}+x_{32}+x_{33}=1 ; \\
x_{i j} \in\{0,1\}, i=1,2,3,4 \text { and } j=1,2,3
\end{gathered}
$$

Solving problem (8.3) we get the optimal assignments $W_{1} \rightarrow J_{3}, W_{2} \rightarrow J_{1}, W_{3} \rightarrow J_{2}$. Also proceeding by the Hungarian method, the optimal assignments are $W_{1} \rightarrow J_{2}$, $W_{2} \rightarrow J_{1}, W_{3} \rightarrow J_{3}$. Now, for optimal assignments $W_{1} \rightarrow J_{3}, W_{2} \rightarrow J_{1}, W_{3} \rightarrow J_{2}$ the minimum assignment cost in terms of IF is $(8,15,25)(7,15,30)+(6,18,25)(5,18,28)+(2,11,14)$ $(1,11,19)$, that is, $(16,44,64)(13,44,77)$ and for optimal assignments $W_{1} \rightarrow J_{2}$, $W_{2} \rightarrow J_{1}, W_{3} \rightarrow J_{3}$ the minimum assignment cost in terms of IF is $(12,14,16)$ $(11,14,28)+(6,18,25)(5,18,28)+(6,12,15)(5,12,20)$, that is, $(24,44,56)(21,44,76)$. More effectively, in reality the assignment cost cannot be taken as fixed/precise one. So, in this chapter we have solved an AP considering each cost of the assignment is IF. For this purpose, to solve the AP with IF cost we have used the ranking function method defined in Definition 8.8 For the IF optimal costs $(16,44,64)(13,44,77)$, we have obtained $\rho((16,44,64)(13,44,77))=31.95$ and $\rho((24,44,56)(21,44,76))=33.59$. Using, Theorem 8.2, we have seen that $\rho((16,44,64)(13,44,77))<\rho((24,44,56)$ $(21,44,76))$; hence, $(16,44,64)(13,44,77)$ is the minimum assignment cost.

\subsection{Conclusion}

In this chapter, AP with IF assignment cost/time is considered and its solution procedure has been established. AP is one of the most essential problems in decisionmaking. In everyday situations, parameters of the AP are imprecise. The AP with IF cost is more reasonable than the AP with precise parameters because most of the 
real-life events are from uncertain domain. The proposed procedure presented here is very effortless and easy for implementation. This solution procedure is based on IF representation of assignment parameters and ranking measure of IF parameters. Here, a newly developed ranking measure based on centroid concept has been used. Then, the AP has been transformed to an AP whose parameters are crisp/precise valued and solved by the existing Hungarian method/linear programming method. Lastly, an IFAP has been solved, and evaluated results have been conferred and measured. It may be asserted that the entire solution procedure set out in this chapter can be put in to solve other realistic decision-making problems in the near future demanding IF parameters.

\section{References}

[1] Balinski M.L. (1986) A competitive (dual) simplex method for the assignment problem, Math Program, 34(2), 125-141.

[2] Barr R.S., Glover F., \& Klingman D. (1977) The alternating basis algorithm for assignment problems, Math Program, 13(1), 1-13.

[3] Huang M.S. \& Rom W.O. (1980) Solving the assignment problem by relaxation, Operations Research, 28(4), 969-982.

[4] Kuhn H.W. (1955) The Hungarian method for assignment problem, Naval Research Logistic Quarterly, 2, 83-97.

[5] Eberhardt S.P., Daud T., Kerns A., Brown T.X., \& Thakoor A.P. (1991) Competitive neural architecture for hardware solution to the assignment problem, Neural Networks, 4(4), 431-442.

[6] Avis D. \& Devroye L. (1985) An analysis of decomposition heuristic for assignment problem, Operations Research Letter, 3(6), 279-283.

[7] Zadeh L.A. (1965) Fuzzy sets, Information and Control, 8(3), 338-352.

[8] Bellman R.E. \& Zadeh L.A. (1970) Decision making in a fuzzy environment, Management Science, 17, 141-164.

[9] Sakawa M., Nishizaki I., \& Uemura Y. (2001) Interactive fuzzy programming for two-level linear and linear fractional production and assignment problems: a case study, European Journal of Operational Research, 135, 142-157.

[10] Chen M.S. (1985) On a fuzzy assignment problem, Tamkang Journal, 22, 407-411.

[11] Huang L.S. \& Zhang L.-P., Solution method for fuzzy assignment problem with restriction on Qualification. Proceeding of Sixth International Conference on Intelligent Systems Design and Applications (ISDA06), 2006.

[12] Liang-hsuan C. \& Hai-wen L. (2007) An extended assignment problem considering multiple inputs and outputs, Applied Mathematical Modeling, 31, 2239-2248.

[13] Majumdar J. \& Bhunia A.K. (2007) Elitist genetic algorithm for assignment problem with imprecise goal, European Journal of Operational Research, 177, 684-692.

[14] Ye X. \& Xu J. (2008) A fuzzy vehicle routing assignment model with connection network based on priority-based genetic algorithm, World Journal of Modeling and Simulation, 4, 257-268.

[15] Thorani Y.L.P. \& Ravi Shankar N. (2013) Fuzzy assignment problem with generalized fuzzy numbers, Applied Mathematical Sciences, 71(7), 3511-3537. 
[16] Chen M.S. \& Chen J.H. (2009) Fuzzy risk analysis based on the ranking generalized fuzzy numbers with different heights and different spreads, Expert System Applications, 36, 6833-6842.

[17] Rommelfanger H.J. (2004) The advantages of fuzzy optimization models in practical use, Fuzzy Optimization and Decision Making, 3(4), 295-309.

[18] Kar S., Basu K., \& Mukherjee S. (2014) Solution of generalized fuzzy assignment problem with restriction on costs under fuzzy environment, International Journal of Fuzzy Mathematics and Systems, 4(2), 169-180.

[19] Thangavelu K., Uthra G., \& Umamageswari R.M. (2016) Solution of fuzzy assignment problem with ranking of generalized trapezoidal fuzzy numbers, International Journal of Pure and Applied Mathematics, 106(6), 9-16.

[20] Andal S., Murgesan S., \& Ramesh B.K. (2016) A novel approach to minimum cost flow of fuzzy assignment problem with fuzzy membership functions, Engineering, Science and Technology: an International journal, 6(2), 85-88.

[21] Pramanik S. \& Biswas P. (2012) Multi-objective assignment problem with generalized trapezoidal fuzzy numbers, International Journal of Applied Information Systems, 2(6), $13-20$.

[22] Mukherjee S. \& Basu K. (2010) Application of fuzzy ranking method for solving assignment problem with fuzzy costs, International Journal of Computational and Applied Mathematics, 5359-5368.

[23] Sahoo L. \& Ghosh S. (2017) Solving assignment problem with linguistic costs, Journal of New Theory, 17, 26-37.

[24] Atanassov K. (1986) Intutionistic fuzzy sets, Fuzzy Sets and Systems, 20, 87-96.

[25] Jana B. \& Roy T.K. (2007) Multi-objective intuitionistic fuzzy linear programming and its application in transportation model, Notes Intuitionistic Fuzzy Sets, 13(1), 34-51.

[26] Roy S.K., Ali E., Verdegay J.L., \& Das S. (2018) New approach for solving intuitionistic fuzzy multi-objective transportation problem, Sadhana, 43(3), 1-12.

[27] Kumar P.S. \& Hussain R.J. (2016) Computationally simple approach for solving fully intuitionistic fuzzy real life transportation problems, International Journal of System Assurance Engineering and Management, 7(1), 90-101.

[28] Arun Prakash K., Suresh M., \& Vengataasalam S. (2016) A new approach for ranking of intuitionistic fuzzy numbers using a centroid concept, Mathematical Sciences, 10(4), 177-184. 